\title{
Brazilian Society of Anesthesiology Recommendations: New Approach to Old Challenges
}

The Brazilian Society of Anesthesiology (SBA) develops and adds one more tool to its continuous and relentless struggle to comply with its institutional mission of bringing together anesthesiologists in Brazil, promoting continuous training, technical and scientific updating, and implementation of advocacy work, as well as fomenting the commitment of the specialty with the medical community and society in general. That is the elaboration, publication and dissemination of evidence-based recommendations on a specific topic in anesthesiology. The purpose of the institution is to provide basis for the anesthesiologist to perform the most effective practices to ensure the quality and safety of medical care, and reduce morbidity and mortality related to all activities that permeate the physician assignments.

The first work done with this objective is published in this issue of RBA, entitled Recommendations of the Brazilian Society of Anesthesiology for Safety in Regional Anesthesia ${ }^{1}$. The SBA's project Recomendações (Recommendations) meets the goals of the Society's current strategic plan. The choice of theme is consistent with the trends of national and global context in which SBA is inserted, of reinforcing actions aimed the quality and safety of anesthesia.

Historically, SBA has sought to remain aligned to important institutions in the search for indicators and evidence of good practices associated with better outcomes of medical activities. It participated in the creation and has collaborated since then with the Projeto Diretrizes da Associação Médica Brasileira (Brazilian Medical Association - AMB Guidelines Project), which introduced in Brazil the sieries of medical guidelines, referral to specialists, health care professionals and an entire related institutional and social chain. The AMB guidelines, developed in conjunction with the medical societies that have an interface with a theme, are diagnostic, therapeutic and preventive guidelines based on scientific evidence, combining medical information in order to standardize procedures that assist the physician's reasoning and decision making.

More recently, SBA has become a signatory to the Declaration of Helsinki of Quality and Safety in Anaesthesia ${ }^{2}$. The Declaration is an international cooperation project originated during the European Congress of Anesthesiology in the city of Helsinki in June 2010. The project statement describes a sequence of points to be followed by the constituent units, with a commitment to reduce rates of morbidity and mortality related to anesthesia. That should be carried out through the development, dissemination and implementation of standard measures aimed to achieve better results by the professionals in the fields of anesthesiology, critical care, emergency as well as pain medicine. They also include all processes and periopera- tive situations in or outside of hospitals, which should bring in higher quality and patient safety in the anesthesia care team.

With nearly ten thousand members, one of the largest societies of anesthesiology at the World Federation of Societies of Anesthesiologists (WFSA), it is imperative for SBA to educate ourselves and become aware of the need to acquire skills for research and publication. Moreover, there must be systematic registration of complications and non-compliance of our activities as anesthesiologists. Therefore, it is essential the construction of integrated reporting, with voluntary and systematic commitment of the professionals. In a pioneer way, the SBA's Comissão de Ensino e Treinamento (Committee of Education and Training) released to its Centros de Ensino e Treinamento (Education and Training Centers - CET) and specialized physicians (SP), the online report of activities of the centers and the logbook, for online description of each of anesthetic practice of SP, contributing to the birth of a national database of a homogeneous population, as members of a CET/SBA. The Comissão de Qualidade e Segurança em Anestesia (Committee of Quality and Safety in Anesthesia) works on planning a system for deployment, organization and monitoring of security protocols, suggesting improvements and reviews of medical care processes, in addition to expanding and improving the tools already in use, providing educational activities and tools for qualification and certification.

The consistency of information from the registration progra$\mathrm{ms}$ originated from our pool of anesthesia adds more weight and volume to the participation of the Brazilian anesthesiology in future recommendations of the SBA. Consequently, we are exposing to the world scientific community the results of our work. The expected return is a greater exchange of experiences, improvement of current practices and establishment of benchmarks for each area of anesthesiology.

Following this direction, always by the light of ethics and the best current scientific evidence, SBA makes a commitment and stands for the responsibility to research, prepare and recommend the use of drugs, equipment, techniques and anesthetic approaches. RBA will always be the first and largest vehicle to carry and present these recommendations.

Who benefits from all this? Everyone. The professionals, the institutions, the industry committed to the standards of excellence and above all our target audience, the patients in our care.

Nádia Maria da Conceição Duarte President of the Brazilian Society of Anesthesiology $S B A-2011$ 